\title{
Effect of Bird-strike on Sandwich Composite Aircraft Wing Leading Edge
}

\author{
*B. Arachchige, ${ }^{\wedge} \mathrm{H}$. Ghasemnejad ${ }^{1},{ }^{\wedge}$ M. Yasaee \\ 'Centre for Structures, Assembly and Intelligent Automation, Cranfield University, UK \\ ${ }^{*}$ Rolls Royce, Derby, UK
}

\begin{abstract}
.
In this paper, a parametric numerical study is performed on the sandwich composite leading edge to analyse the effect of skin thickness, layups, impact velocities to compare the performance of the two different reinforcements within sandwich leading-edge structures. The detailed numerical analysis of a composite leading edge reinforced with honeycomb and foam is developed using explicit finite element software, LS-DYNA. Initially, the study proposes the most suitable equations of state for impact on the metallic leading edge for different bird geometries made from Lagrangian and SPH methods. All the numerical results are verified with available experimental data in the literature. The results will deliver a cost-efficient and accurate numerical model which assists aircraft designers in deciding the combination of design variables resulting in improved impact resistance for sandwich aircraft structures under soft body impacts.
\end{abstract}

\section{Keywords.}

Bird strike, Lagrangian, Sandwich, Leading Edge, LS-DYNA, SPH

\footnotetext{
${ }^{1}$ Corresponding Author: email: Hessam.Ghasemnejad@cranfield.ac.uk Tel.:+44 (0) 1234754395.

Centre for Structures, Assembly and Intelligent Automation, Cranfield University, MK43 0AL, UK.
} 


\section{1- Introduction}

Modern jet aircraft are carrying an increased amount of passengers and therefore a small amount of damage can lead to a catastrophic chain of events. Hence, it is critical to ensure that the different structural parts such as compressor blades, wing/tail leading edges, wind-shield can resist high energy impacts and guarantee a safe landing of the aircraft after a bird strike. Experimental tests for a bird-strike certification are very expensive and time-consuming, therefore, an accurate and reliable analysis tool that predicts the structural response of bird and target is required. Finite element simulation is a widely used tool to evaluate numerous structural design approaches that reduce structural weight and minimise risks of not meeting aircraft birdstrike design standards. Non-linear finite element codes are capable of predicting loads and deformations of both bird and aircraft structure with reasonable levels of accuracy. According to the International Bird Strike Committee database, 55 fatal accidents have occurred between 1912 and 2009, where 108 aircraft were damaged and 277 passengers were killed [1]. Birdstrike is a high-velocity impact where materials with huge variations in material property come into contact, thus resulting in non-linear material behaviour, high strain rates and extremely large deformation. The use of composite materials in aircraft structures construction has increased rapidly due to its attractive properties such as high stiffness to weight ratios, high energy absorption capabilities, corrosion and fatigue resistance, etc. However, their major drawback is their vulnerability against transverse impact loads which leads to interlaminar (delaminations) and intralaminar (fibre/matrix) failure modes, thus severely reducing the load-carrying ability of these structures. A birdstrike impact event can lead to these transverse impact loads. 
During the early days when finite element methods were not available, Barber and Wilbeck [2, 3] pioneered experimental impact tests on a rigid plate. Birds with different body shapes and weights were used. They found that peak pressures generated at the centre of the plate were independent of the bird shape, but was proportional to the square of the impact velocity. Researchers have used three different well-established approaches to discretize the bird model: (a) Lagrangian, (b) Arbitrary Lagrangian-Eulerian (ALE) and (c) Smooth Particle Hydrodynamics (SPH). These approaches are explained in detail in section 2 of the paper.

Hedayati and Ziaei-Rad [4] performed numerical modelling of birdstrike with a bird geometry similar to the real bird. They studied the effect of bird orientation on pressure profiles during impact and concluded that impact from bird's bottom side is the most dangerous scenario, while the tail side impact is the least dangerous. Guida et al. [5] conducted a numerical investigation of the capability of a fibre metal sandwich wing leading-edge construction subjected to bird impact using Lagrangian and SPH approach. They proved that both methods predicted failure mechanisms similar to the experimental test and reproduced the dynamic response of the impact event in detail. They also stated that the SPH approach produced a more realistic global deformation compared to the traditional Lagrangian method. Lavoie et al. [6] compared Lagrangian, ALE and SPH numerical bird modelling approaches with experimental data. They proved that ALE and SPH models compare well with theoretical predictions. They also confirmed that the pressure, mass loss and radial pressure distribution through Lagrangian approach are inaccurate. Heimbs [7] preformed numerical modelling and simulation of highvelocity impact loads from soft body projectiles on composite structures. He showed that the preloading of the composite structure has a significant impact on its structural response. A stacked shell modelling technique was used with cohesive elements for interlaminar failure modelling and Hashin failure criterion was used in predicting intralaminar failure. Numerical predictions compared well with experimental results. Goyal et al. [8] developed a smoothed 
particle hydrodynamic approach in LS-DYNA and compared results with a Lagrangian model and experimental data. Their analysis showed that the mesh density of the SPH bird model has a strong influence on the peak impact pressure. They also proved that the SPH model performed well for angle impacts with a 7\% difference in maximum normal deflection when compared to experimental data. Kim et al. [9] studied bird impact on static single composite blades and a full rotating fan stage using ALE and SPH approach. It was concluded that the Hugoniot region of the impact was less significant than the stagnation pressure over the complete impact event. They discovered that in addition to direct impact damage, blades were more likely to be severely damaged by colliding with neighbouring blades. Liu et al. [10] performed experiments of bird impact on flat plates and explicit finite element analysis through PAM-CRASH software. They stated that the elastic-plastic material model with a defined failure strain is best suited for bird strike simulation at low impact velocities, the isotropic elastic-plastic hydrodynamic solid model for intermediate impact velocities and the SPH method coupled with Murnaghan equation of state is best suited for high-velocity bird impacts. Experimental and numerical simulation of bird impacts on aluminium foam-based sandwich panels were carried out by Hanssen et al. [11]. A continuum damage mechanics based constitutive model was used to describe the behaviour and failure of aluminium cover plates. In modelling the foam core, a pressure-sensitive constitutive model coupled with a failure criterion on maximum volumetric strain was adopted. This model was capable of determining the minimum foam core thickness to avoid penetration of the bird. Liu et al. [12] designed a novel tail leading edge structure using finite element method coupled with SPH approach to simulate bird strike. They introduced a triangular reinforcement component that enhanced performance when subjected to a birdstrike. Experimental tests and finite element predictions showed that this reinforcement component reduces the deformation and damage of tail leading edge. Smojver and Ivancevic [13] numerically predicted bird strike induced damage on aircraft inboard flap. Their results 
showed that damage critically depends on the bird mass and deflection of the applied velocity vector. Honeycomb and foam have been used in this paper to reinforce the composite leading edge against soft body impacts. The effect of impact velocity, skin thickness, layups, equations of state on impact response is studied. Initially, a numerical model is developed for the metallic leading edge and compared with experimental results for different bird geometries and equations of state. The orientation of the different layers in a composite laminate plays a vital role in the impact response. However, more recent studies related to studying bird impact response of reinforced composites are mainly focused on the laminate orientations of the composite skin without core and sandwich structures [14 - 17]. Therefore, this paper aims to fill that gap in research and propose advanced anisotropic structures to study the impact response of composite skins with honeycomb and foam core reinforcements under bird impacts.

\section{2- Numerical Bird Modelling Techniques}

First approaches of bird strike modelling involved representing the bird as a pressure pulse on the structure. The main assumption behind this theory was due to a bird largely consists of water and therefore be represented as a water jet. Currently, there are three main modelling methods: (a) Lagrangian, (b) Arbitrary Lagrangian-Euler (ALE) and (c) Smooth particle hydrodynamic (SPH) method [18 - 19], Figure 1.
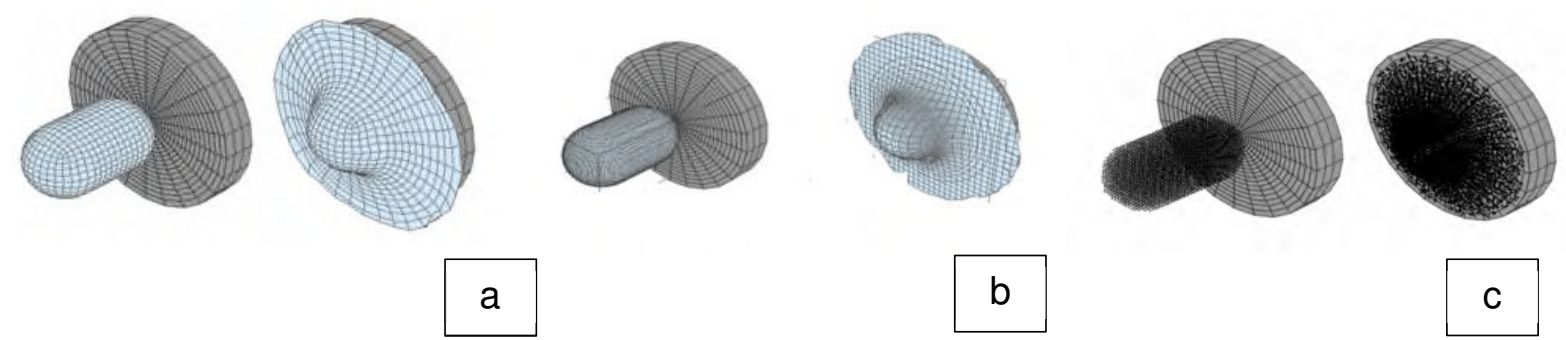

Figure 1. Soft body impact models. (a) Lagrangian (b) ALE and (c) SPH [18 - 19]. 


\section{3- Finite Element Modelling Techniques}

Initially, three models are developed, first hemispherical Lagrangian, flat-ended Lagrangian, and hemispherical SPH (Figures 2 and 3) respectively. Material properties used in the experimental studies [2,3] are given in Tables 1 and 2.

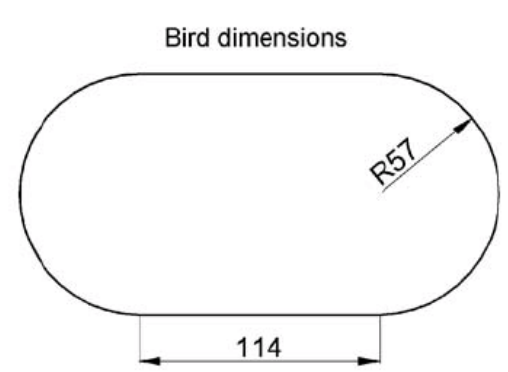

(a)

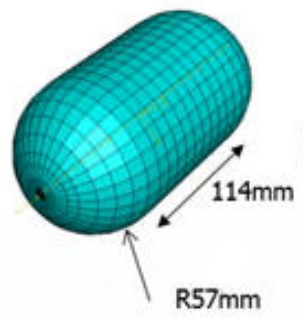

(b)

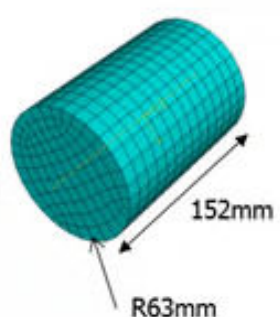

(c)

(d)

Figure 2. Bird geometries. (a) Bird dimensions (b) Hemispherical Lagrangian (c) Flat ended Lagrangian (d) Hemispherical SPH.

Table 1. Material properties of the bird in Lagrangian and SPH models [2, 3].

\begin{tabular}{cc}
\hline Property & Value \\
\hline Mass & $1.82 \mathrm{~kg}$ \\
\hline Average density & $950 \mathrm{~kg} / \mathrm{m}^{3}$ \\
\hline Cut-off pressure & $0.01 \mathrm{MPa}$
\end{tabular}

Table 2. Material properties of target plate $[2,3]$.

\begin{tabular}{cc}
\hline Property & Value \\
\hline Elastic Modulus & $205 \mathrm{GPa}$ \\
\hline Density & $7800 \mathrm{~kg} / \mathrm{m}^{3}$ \\
\hline Poisson's ratio & 0.29 \\
\hline Diameter & $0.2 \mathrm{~m}$ \\
\hline
\end{tabular}




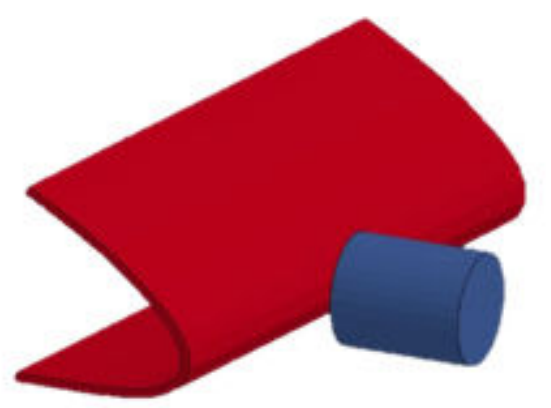

(a)

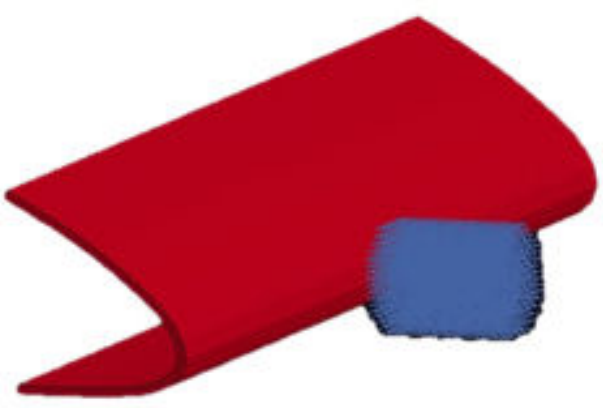

(b)

Figure 3. Bird impact on metallic leading edge (a) Flat ended Lagrangian (b) Hemispherical SPH.

\section{4- Material Modelling for Target Leading Edge}

\subsection{Johnson Cook Material Model}

Johnson-Cook material model is used to model high rate deformation of many materials including metals. In LS-DYNA, this material model is MAT_015_Johnson_Cook. The flow stress in Johnson-Cook material model is defined by:

$\sigma_{y}=\left(A+B \bar{\varepsilon}^{p^{n}}\right)\left(1+C \ln \dot{\varepsilon}^{*}\right)\left(1-T^{*^{m}}\right)$

\begin{tabular}{cc}
\hline Property & Value \\
\hline Young's Modulus & $205 \mathrm{GPa}$ \\
\hline Poisson's Ratio & 0.29 \\
\hline A & $217 \mathrm{MPa}$ \\
\hline B & $234 \mathrm{MPa}$ \\
\hline $\mathrm{n}$ & 0.6428 \\
\hline $\mathrm{C}$ & 0.0756
\end{tabular}

Table 3 Johnson-Cook material properties for steel [20], A: Yield Strength and B: Ultimate Tensile Strength. 


\subsection{Honeycomb Material Model}

This material model is mainly used for honeycomb and foam materials with real anisotropic behaviour. A non-linear elastoplastic material behaviour can be defined separately for all normal and shear stresses. The present study adopts material model MAT_026_HONEYCOMB. Material inputs of this model are shown in Table 4 and Figure 4.

\section{Parameter}

\section{Value}

Density

$198 \mathrm{~kg} / \mathrm{m}^{3}$

Young's modulus of compressed honeycomb

$137 \mathrm{GPa}$

Poisson's ratio of compressed honeycomb

0.33

Relative volume

0.15

Yield stress

$280 \mathrm{MPa}$

Material viscosity coefficient

0.06

Elastic modulus of uncompressed honeycomb in a- direction

$1.45 \mathrm{GPa}$

Elastic modulus of uncompressed honeycomb in b- direction

$1.45 \mathrm{GPa}$

Elastic modulus of uncompressed honeycomb in c- direction

$138 \mathrm{GPa}$

Shear modulus of uncompressed honeycomb in ab plane

$2 \mathrm{GPa}$

Shear modulus of uncompressed honeycomb in bc plane

$20 \mathrm{GPa}$

Shear modulus of uncompressed honeycomb in ca plane

$40 \mathrm{GPa}$

Table 4. MAT_026 material model properties [21]. 


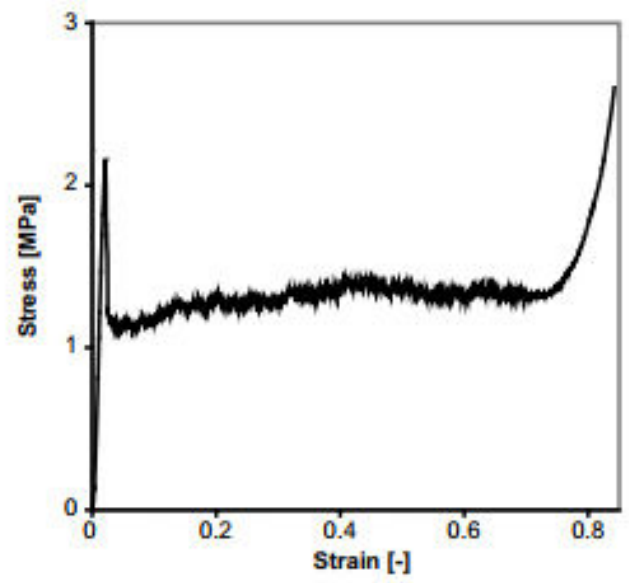

Figure 4. Compressive stress-strain behaviour of HRH 10-1/8-3.0 Nomex honeycomb [22].

\subsection{Composite Material Model}

Material model 54 based on the Chang-Chang failure criteria is used to model laminated composite design in this section. The leading edge is meshed using shell elements with the formulation of Belytschko-Tsay. The element size is $2.5 \times 2.5$ according to the mesh sensitivity analysis in previous work of authors [23]. Material input data is shown in Table 5 and 6.

\section{Parameter}

Value

Longitudinal Young's Modulus $135 \mathrm{GPa}$

Transverse Young's Modulus $10 \mathrm{GPa}$

Poisson's ratio

0.3

Shear modulus

$5 \mathrm{GPa}$

Longitudinal tensile strength

$1500 \mathrm{MPa}$

Transverse tensile strength

$50 \mathrm{MPa}$

Longitudinal compressive strength

$1200 \mathrm{MPa}$

Transverse compressive strength

$250 \mathrm{MPa}$

Shear strength

$70 \mathrm{MPa}$

Table 5. Uni-directional Carbon Fibre properties - Layup $1[0 / 90 / 0 / 90]_{2 s}$ and Layup 2 [0/45/$45 / 90]_{2 s}[24]$. 
This material model in LS-DYNA has been developed to represent properties of isotropic crushable foam which includes optional damping and tensile cut-off stress. In LS-DYNA, material model 63 (MAT_CRUSHABLE_FOAM) represents crushable foam that requires a user-defined curve signifying yield stress versus volumetric strain response of the material (see Figures 5 - 7).

\begin{tabular}{cc}
\hline Description & Value \\
\hline Density & $12.5 \mathrm{~kg} / \mathrm{m}^{3}$ \\
\hline Young's Modulus & $0.022 \mathrm{GPa}$ \\
\hline Damping coefficient & 0.5 \\
\hline
\end{tabular}

Table 6. Material properties for MAT_CRUSHABLE_FOAM [25].

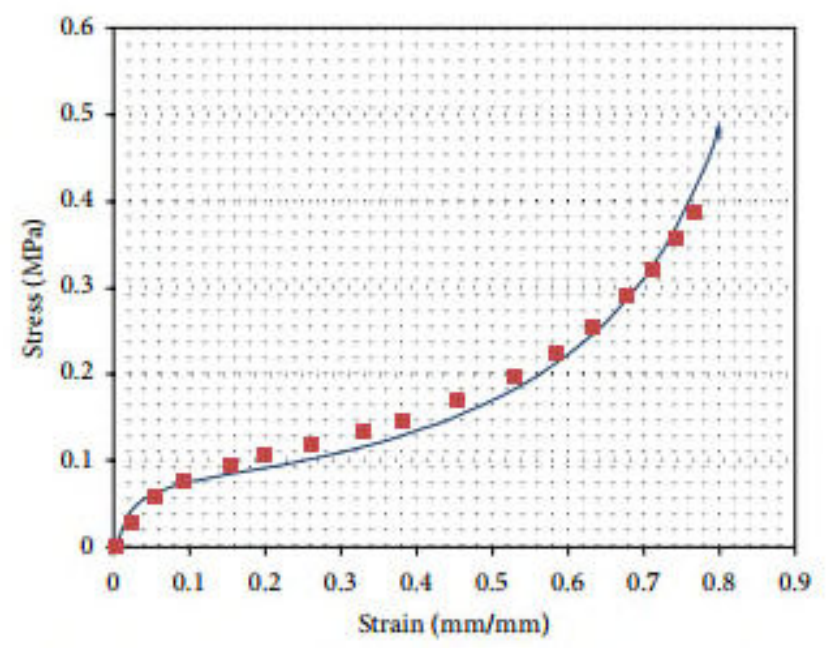

Figure 5. Stress-strain relationship for EPS foam [25]. 

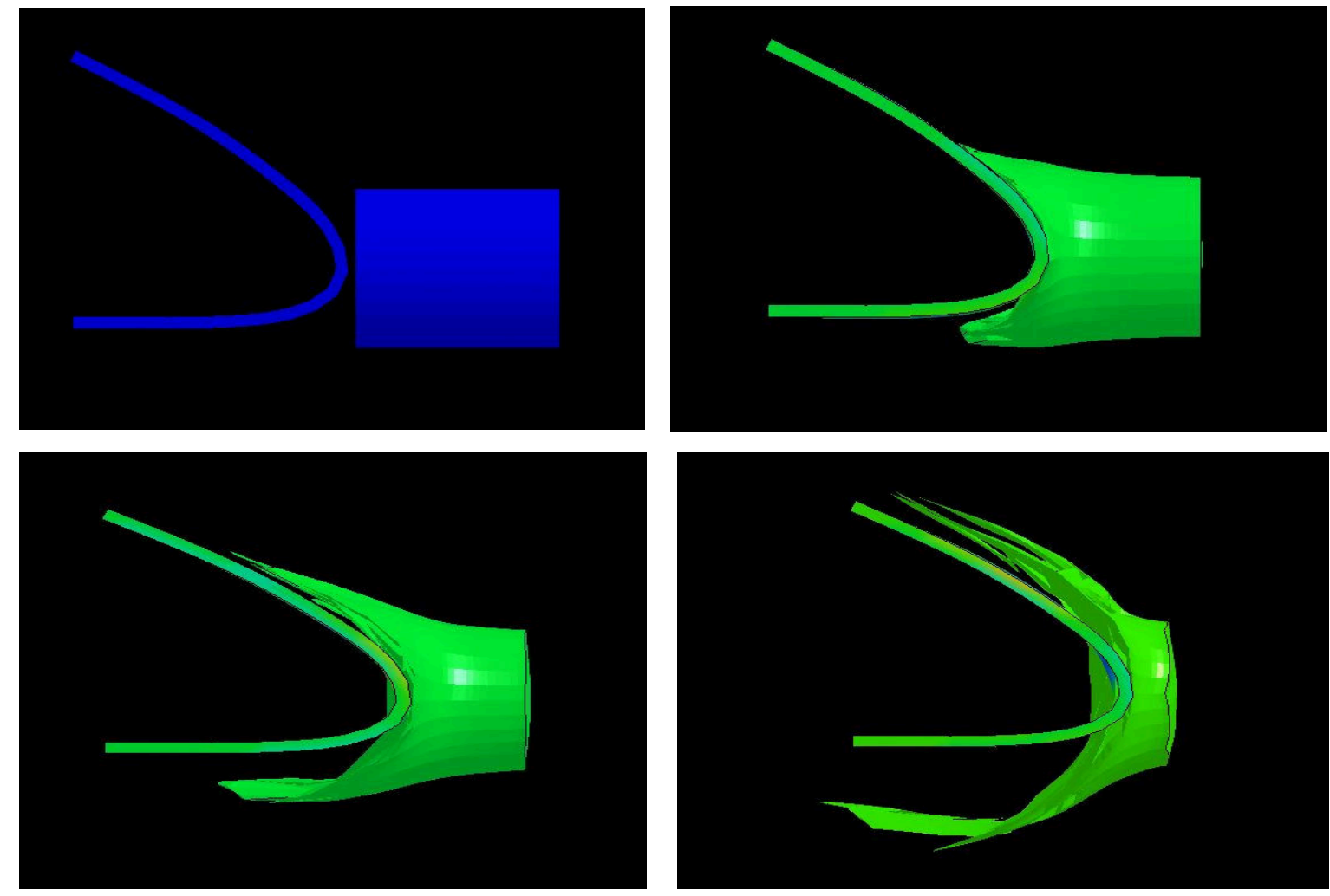

Figure 6. Pressure distribution of Lagrangian bird impact models for the metallic leading edge.
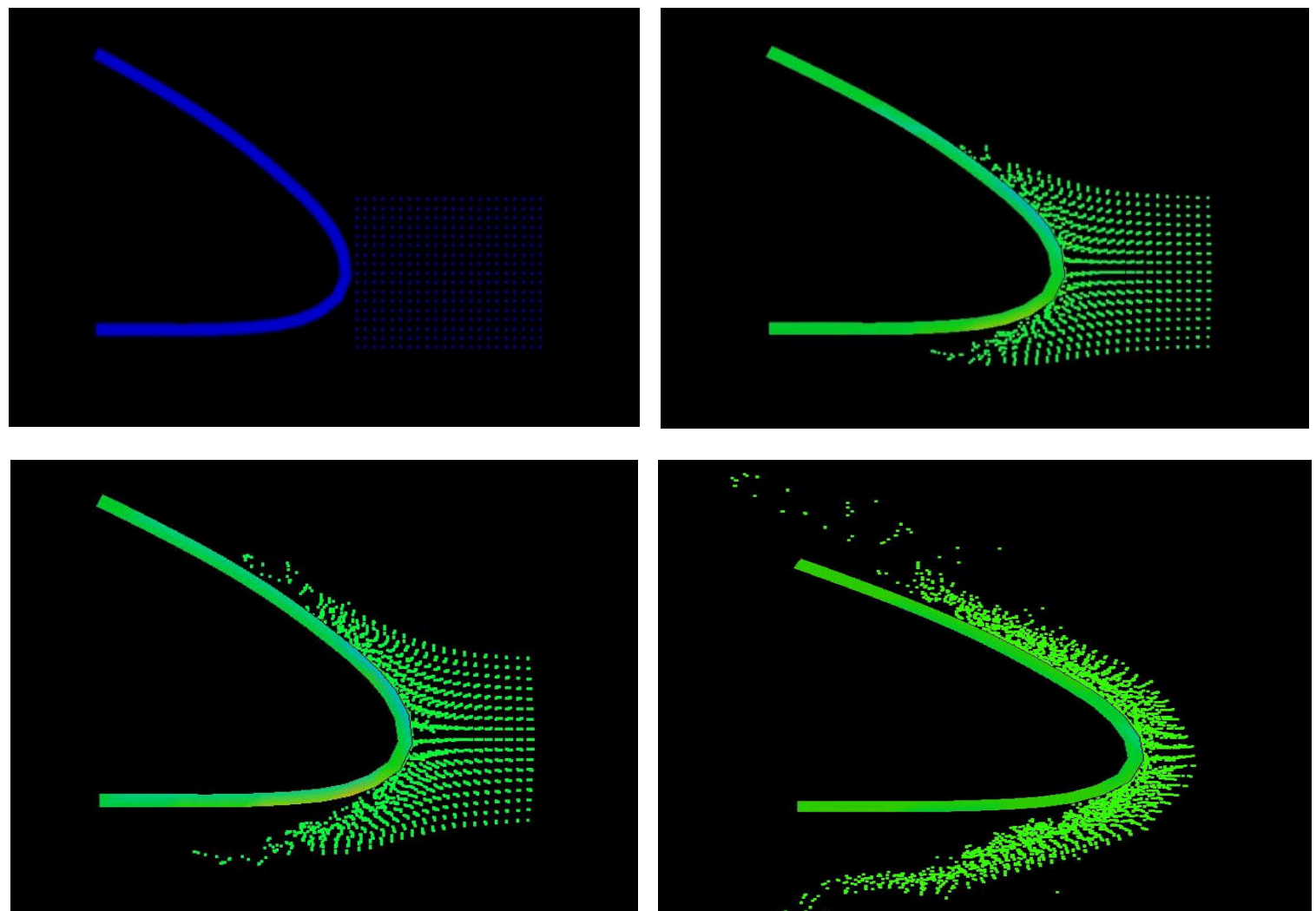

Figure 7. Pressure distribution of SPH bird impact models for the metallic leading edge. 


\section{5- Verification of Numerical Studies}

\subsection{Lagrangian and SPH Bird Model Comparison}

Barber et al $[2,3]$ experimentally investigated bird impact behaviour on the flat panels. Their results showed that the most suitable substitute material for the bird is gelatine, which is mixed by air to obtain a final porosity of $10 \%$ with an average density of $950 \mathrm{~kg} / \mathrm{m}^{3}$. These tests also proved that the most suitable projector shape for impact testing was a cylinder with hemispherical ends and a length-to-diameter ratio of 2 . In the impact tests, several birds and substitutes were fired onto a rigid plate on which four piezoelectric quartz transducers were located along the radius toward the centre of impact. The projectiles were fired at velocities, which were ranged from 100-300 m/s perpendicularly and obliquely at angles of 25 and 45 degrees to the normal of the plate. The results were presented in normalised dimensions of pressure versus time where the pressure was divided by the stagnation pressure and the time of impact. The results were sensible since there is a rise of pressure and then it was stabilised around its stagnation value at around one-third of the impact.

Figure 8 shows a comparison between Lagrangian and SPH methods for the linear equation of state with $10 \%$ porosity and hemispherical ended bird model. The results indicate that the Lagrangian approach over-estimates the Hugoniot pressure. Lagrangian Hugoniot pressure is $54 \%$ higher than the experimental method, whereas the SPH method is $14 \%$. Therefore, it is concluded that the SPH method yields better predictions when compared with the Lagrangian method. 


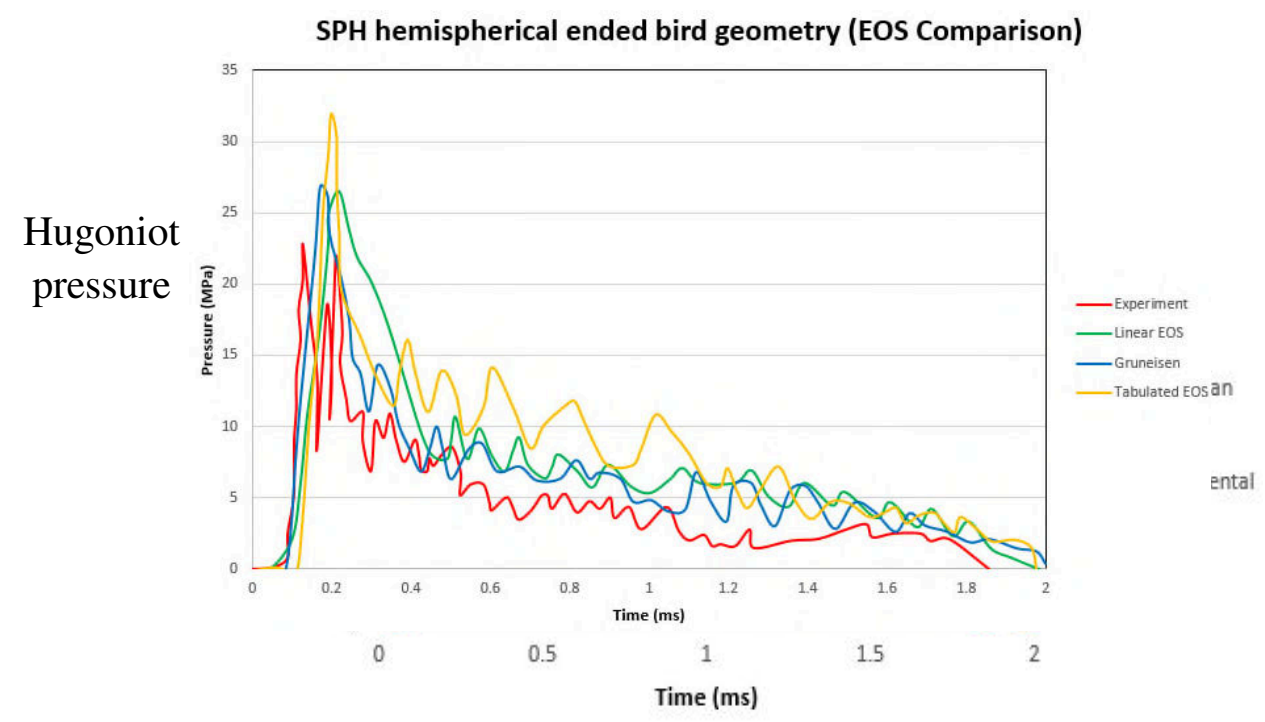

Figure 8. Comparison between Lagrangian, $\mathrm{SPH}$ method and experiment [2, 3].

\subsection{EOS Comparison}

The equation of state (EOS) is one of the governing factors in pressure distribution during bird impact. Comparison of the equation of states for different bird geometries is shown in Figures 9 and 10. It is observed that for the hemispherical ended bird geometry, Gruneisen EOS is the most suitable since it closely matches with experimental Hugoniot pressure. However, for the flat-ended bird geometry, the tabulated EOS seemed to be the more reasonable choice in comparison with other cases.

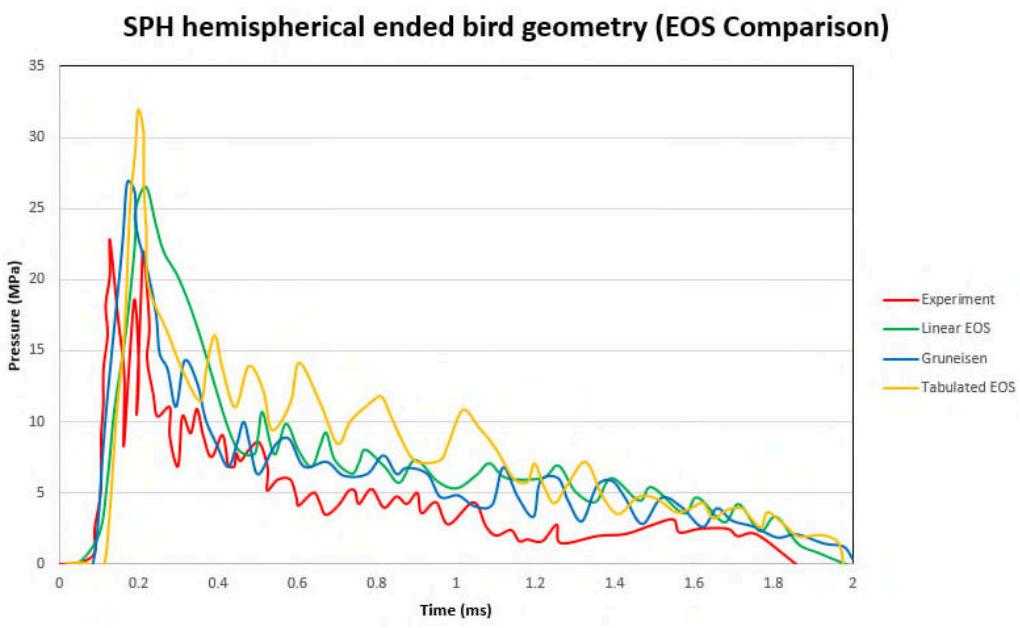

Figure 9. Comparison between hemispherical ended bird geometry and experiment $[2,3]$. 


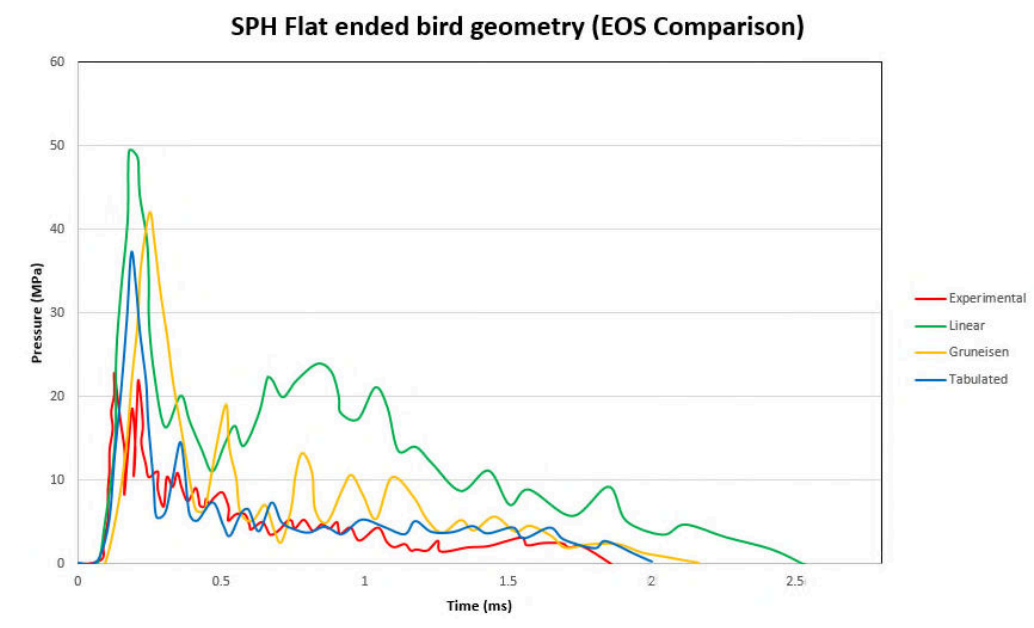

Figure 10. Comparison between flat-ended bird geometry and experiment [2,3].

\section{6- Composite Leading-edge FE Model}

FE models are developed for reinforcing a composite leading edge with honeycomb and foam core. In these models, the leading edge is meshed using shell elements and hemispherical ended bird geometry is used with SPH elements. Gruneisen equation of state has been adopted for all simulations on composite leading edges with and without reinforcement. The thickness of the reinforcement is $5 \mathrm{~mm}$ (see Figure 11). 


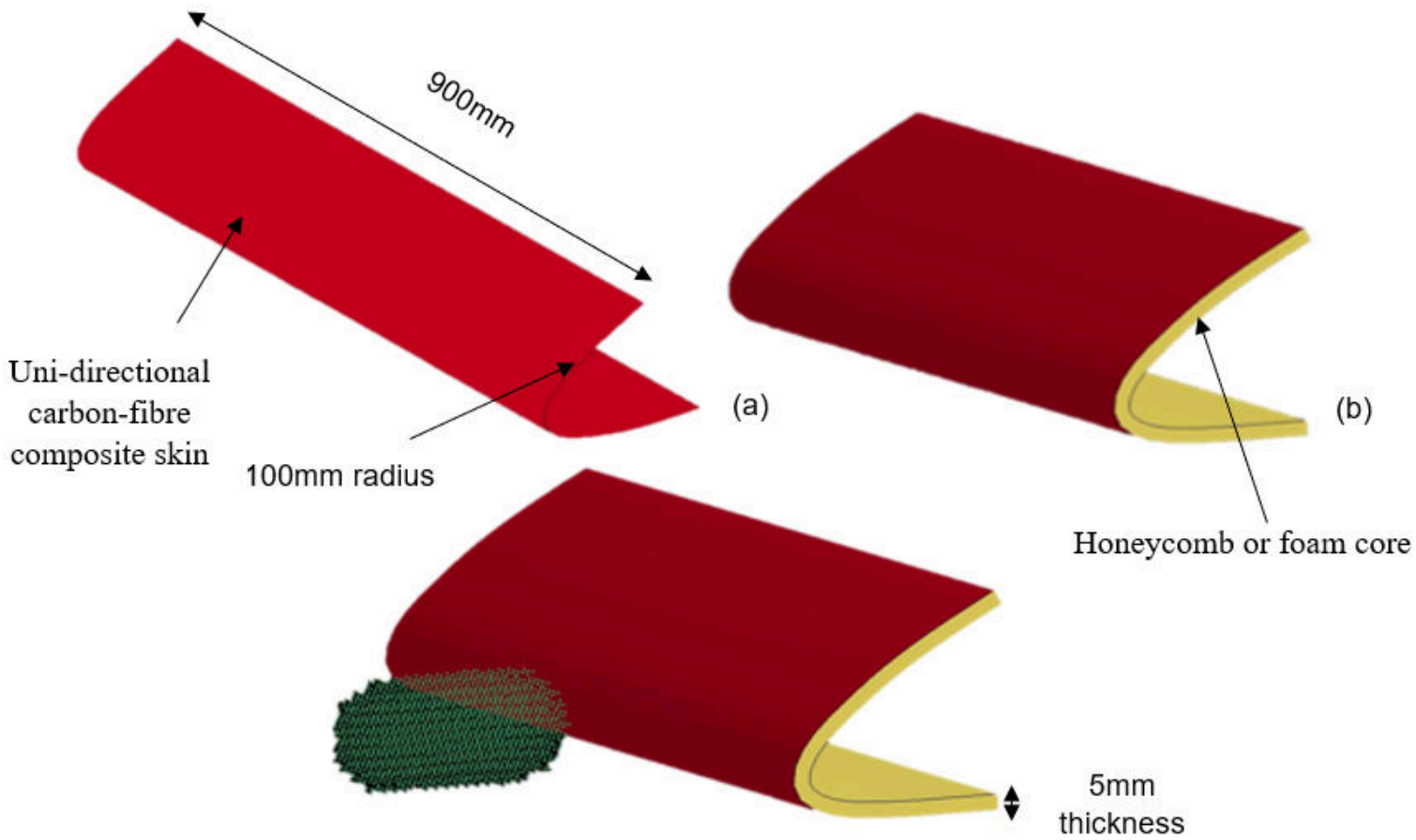

(c)

Figure 11. FE models for the composite leading edge (a) without reinforcement, (b) with honeycomb or foam reinforcement, (c) impact with SPH bird model.

\subsection{Effect of Thickness}

The effect of composite skin thickness is studied on the leading edge without reinforcement. Contact force history of the analysis depicts that increase of thickness of the composite skin enhances the maximum contact force. It is seen that element failure occurs at $2.4 \mathrm{~ms}$ implying that the $0.8 \mathrm{~mm}$ thickness leading edge would not sustain the bird-strike. No failure occurs in the $1.3 \mathrm{~mm}$ leading edge as shown in Figures $12-14$. 

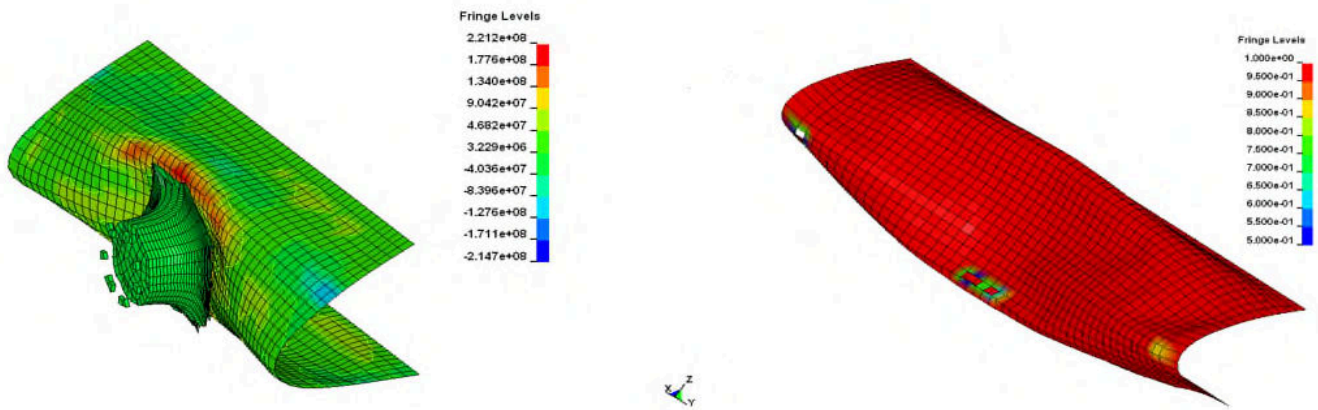

a

b

Figure 12. Impact on $0.8 \mathrm{~mm}$ thickness leading edge, a) pressure distribution and b) effective plastic strain of leading edge.

\section{$1.3 \mathrm{~mm}$ skin thickness of leading-edge}
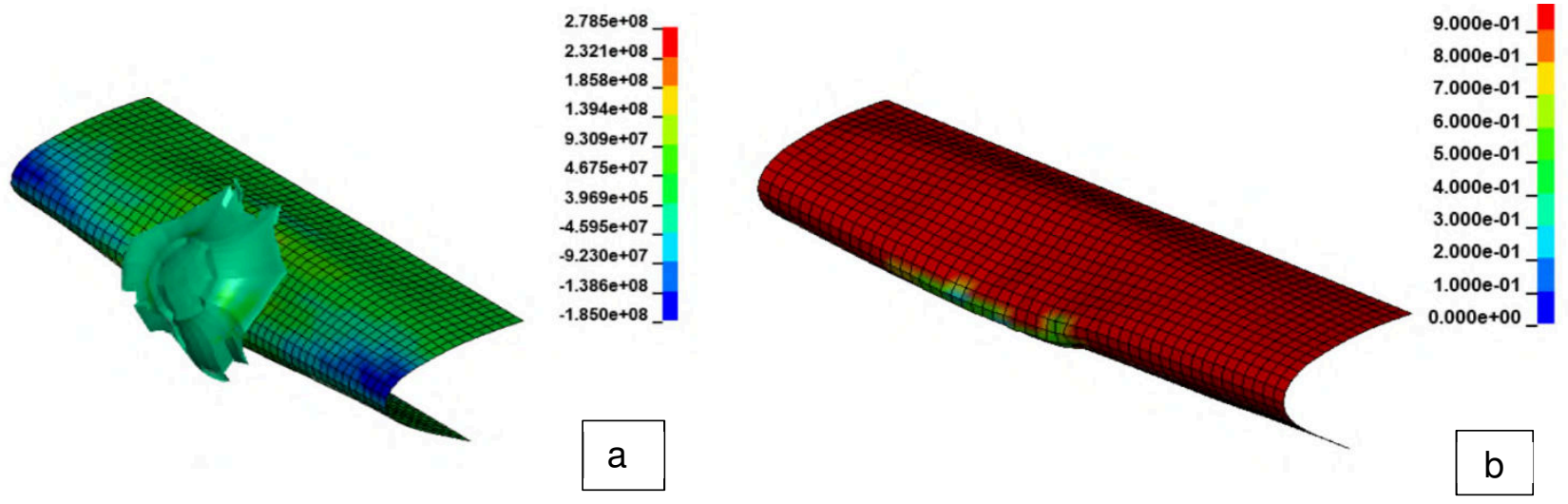

Figure 13. Impact on $1.3 \mathrm{~mm}$ leading edge, a) pressure distribution and b) effective plastic strain of leading edge. 


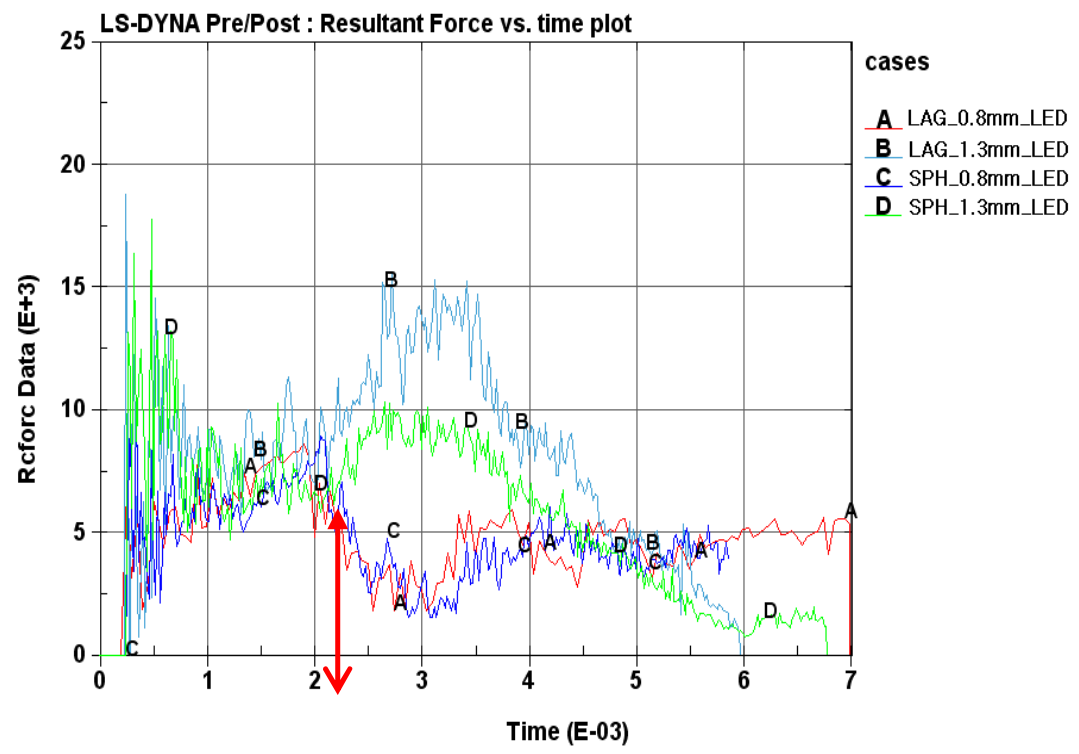

First element failure

Figure 14. Force vs time history plot for both Lagrangian (LAG) and SPH models.

\subsection{Impactor Shape}

The effect of impactor shape is analysed here. A flat-ended and hemispherical nose impactor is considered as shown in Figures 15 - 16.
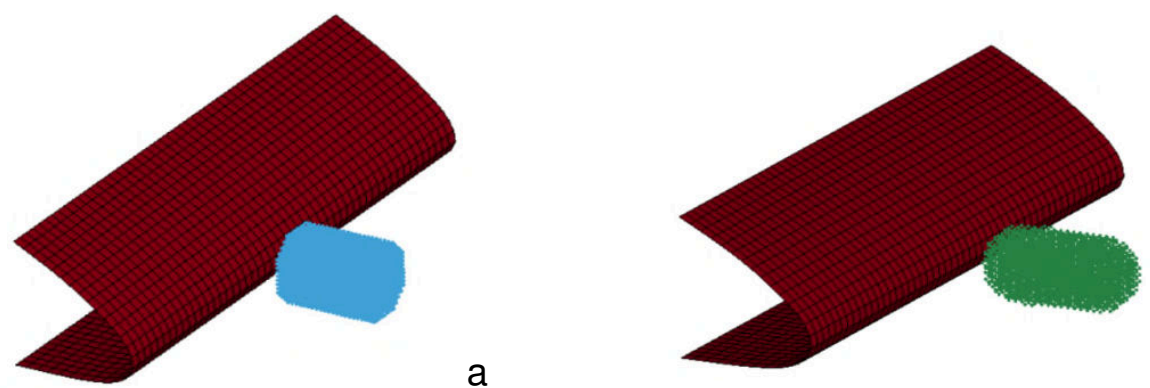

b

Figure 15. a) Flat ended and b) hemispherical nose impactors. 


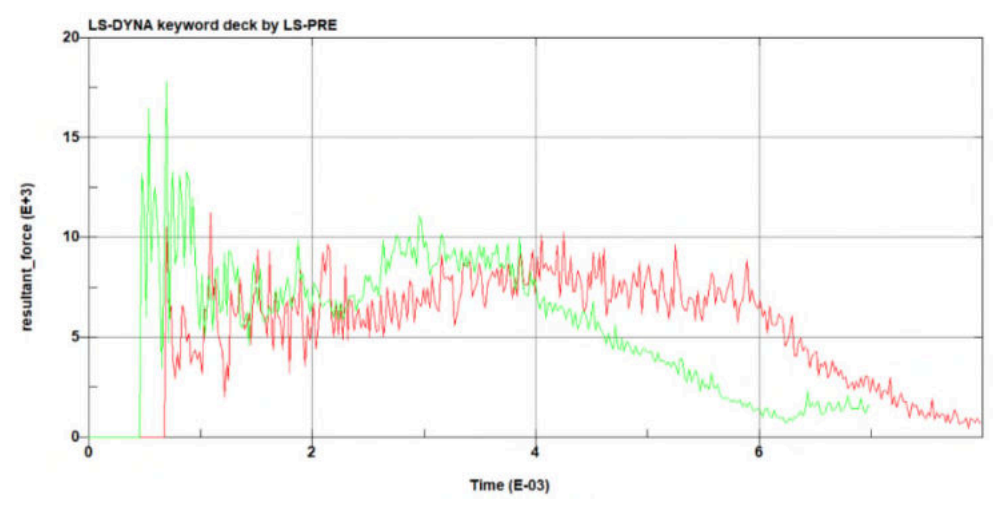

Figure 16. Effect of striker geometry on impact response.

\subsection{Effect of Reinforcement Type}

It is seen that reinforcement plays a vital role in the impact response of the leading edge. For all impact velocities analysed, honeycomb reinforcement showcase superior impact resistance. It is evident that as the velocity increases, the performance of the honeycomb reinforcement increases as well. At $80 \mathrm{~m} / \mathrm{s}$ impact velocity, the maximum contact force of honeycomb reinforcement is $15 \%$ higher than the no reinforcement configuration and $7 \%$ higher than foam reinforcement. When the impact velocity is $116 \mathrm{~m} / \mathrm{s}$, the maximum contact force of honeycomb reinforcement is $16 \%$ higher compared to leading-edge without reinforcement and $8 \%$ greater than foam reinforcement. At an impact velocity of $150 \mathrm{~m} / \mathrm{s}$, the maximum impact force of honeycomb configuration is $20 \%$ higher compared to the no-reinforcement model and $11 \%$ higher than the foam reinforcement (see Figure 17 and 19).

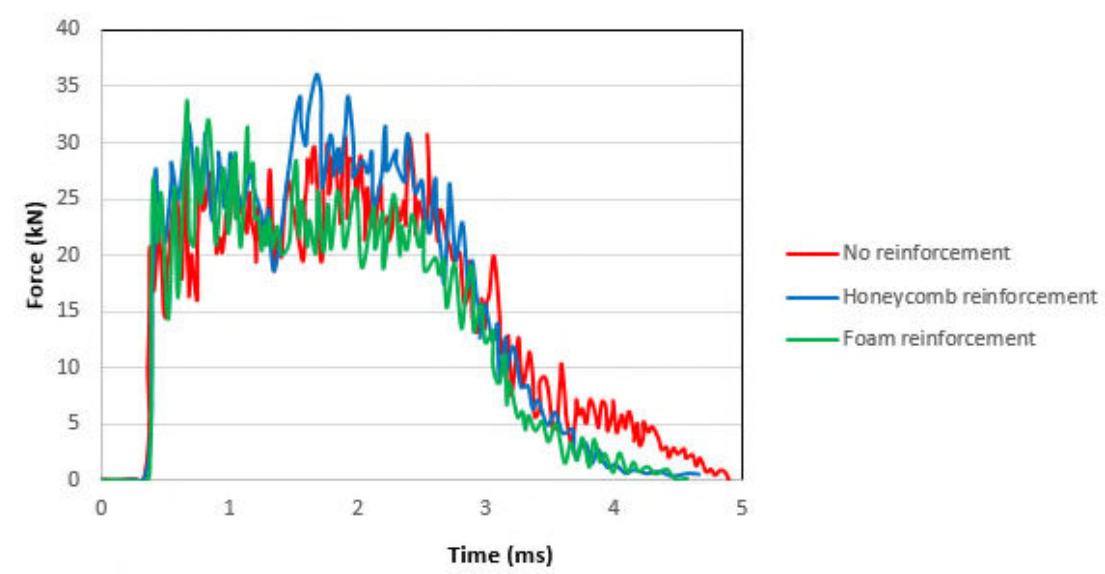

Figure 17. Contact force history (Effect of reinforcement type at $80 \mathrm{~m} / \mathrm{s}$ impact velocity). 


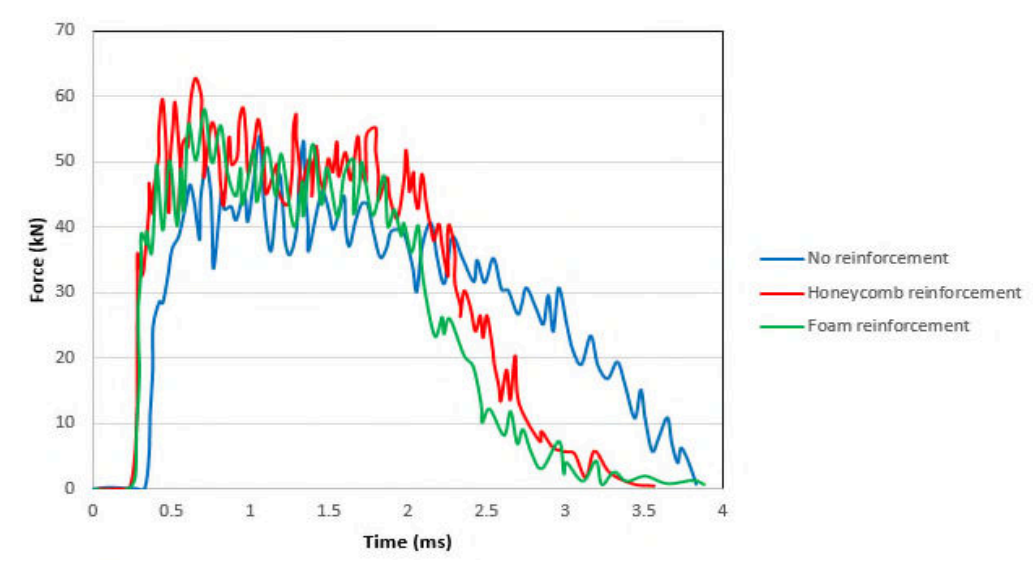

Figure 18. Contact force history (Effect of reinforcement type at $116 \mathrm{~m} / \mathrm{s}$ impact velocity).

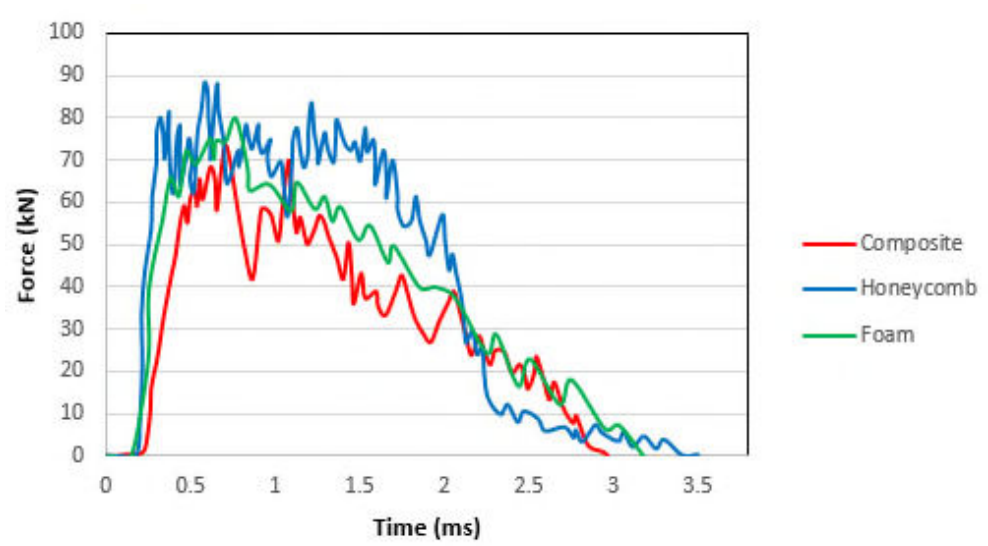

Figure 19. Contact force history (Effect of reinforcement type at $150 \mathrm{~m} / \mathrm{s}$ impact velocity).

\subsection{Effect of Layups}

This numerical analysis investigates that fibre orientations of the composite skin affect the impact performance of the leading edge. Two layups are considered which is the asymmetric cross-ply orientation $\left.\left([0 / 90 / 0 / 90]_{2 s}\right]\right)$ and symmetric quasi-isotropic $([0 / \pm 45 / 90] 2 s)$. It is shown that contact force history plot comparison of the leading edge without reinforcement for both layups are quite similar. Maximum contact force in the cross-ply configuration is only $3 \%$ for the quasi-isotropic layup. This means that changing the layup for a leading-edge without reinforcement has a slight effect on its impact performance. However, the maximum contact force for the quasi-isotropic layup is $10 \%$ higher than the cross-ply layup one. The cross-ply 
layup performs better than the quasi-isotropic layup, where maximum contact force is $7 \%$ greater when it is embedded with foam reinforcement, (see Figures 20 - 22).

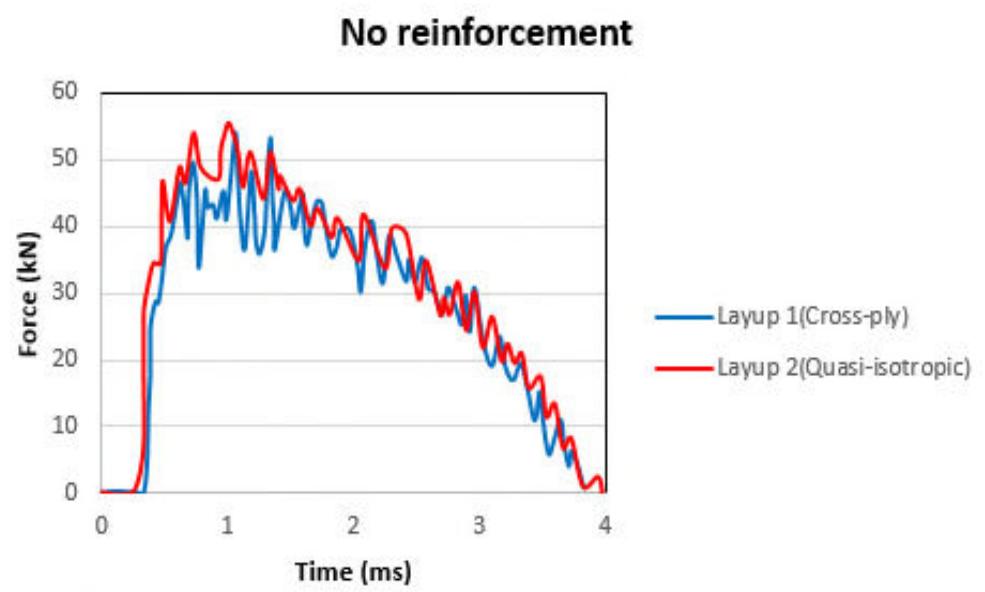

Figure 20. Effect of layups (No reinforcement).

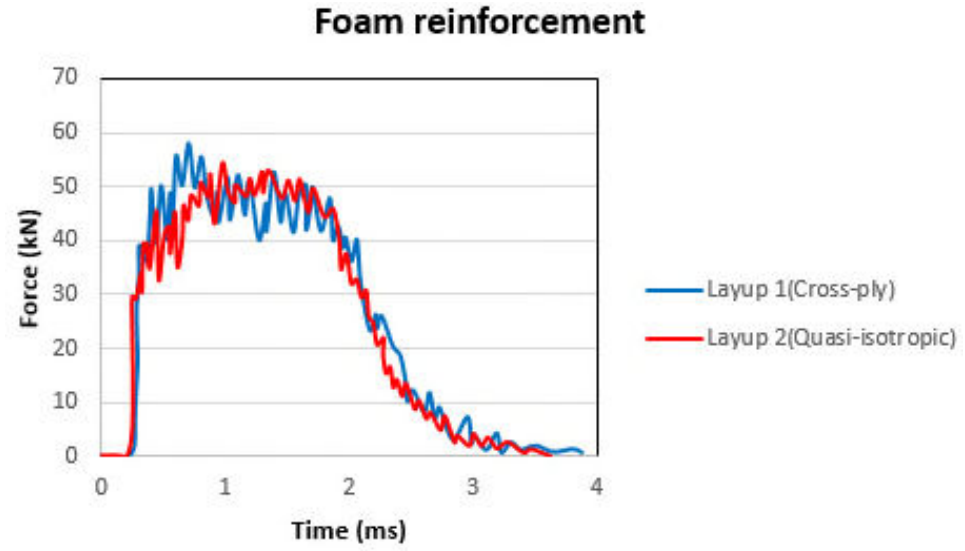

Figure 21. Effect of layups (Honeycomb reinforcement).

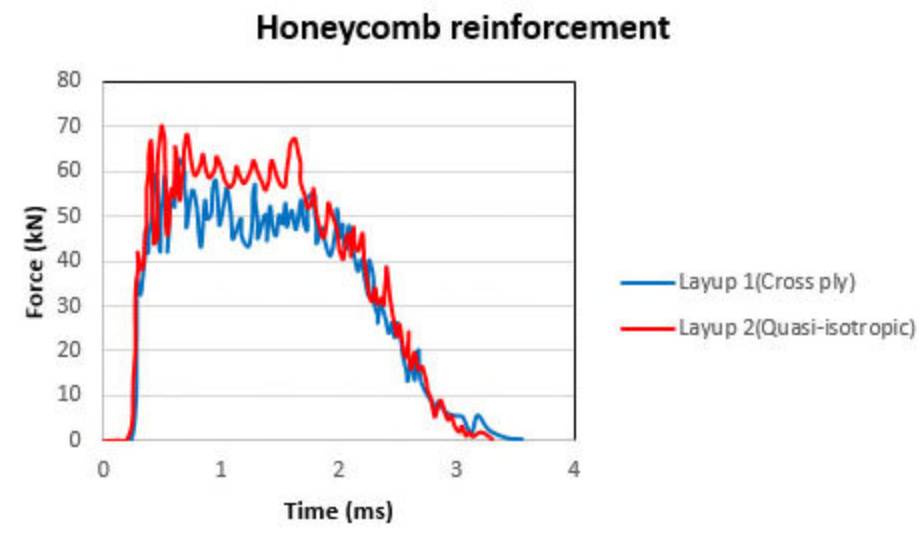

Figure 22. Effect of layups (Foam reinforcement). 


\section{7- Conclusions}

The main aim of this paper was to study the effect of reinforcement of a composite wing leading edge using either honeycomb or foam core on bird impact. Initially, FE models were developed for impact on the metallic leading edge by Lagrangian and SPH methods. The effect of different equations of the state combined with a null material is studied for two different bird geometries. The SPH approach, which showed closer results in comparison with experimental data, was adopted for the later studies. Studies related to EOS comparison were performed and it was observed that Mie-Gruneisen EOS accurately predicted pressure distribution for a hemispherical ended bird while Tabulated EOS (10\% porosity) for a flat-ended bird when compared with experimental results. Honeycomb and foam reinforcement improved the impact resistance of the composite leading edge. The most effective reinforcement was honeycomb where the deflection of the leading edge was $85 \%$ less compared to leading-edge without reinforcement and $45 \%$ less than foam reinforcement at an impact velocity of $80 \mathrm{~m} / \mathrm{s}$. Honeycomb reinforcement also performed well with an increase of velocity. The orientations of the laminate layers of the composite skin too played a vital role in impact response during a bird strike. It was found that a quasi-isotropic layup performed better in terms of damage tolerance when it is compared with a cross-ply layup for the honeycomb reinforcement. However, for the foam reinforcement, the cross-ply layup impact resistance was better in comparison with quasi-isotropic layup. 


\section{8- References}

[1] Hedayati R, Sadighi M, Birdstrike: An experimental, theoretical and numerical investigation. WoodHead Publishing in Mechanical Engineering, September 2015.

[2] Barber J.P., Taylor H.R., Wilbeck J.S.. Characterization of bird impacts on a rigid plate: Part 1. Technical Report AFFDL-TR-75-5, Air Force Flight Dynamics Laboratory; 1975.

[3] Barber, J.P, Taylor H.R., Wilbeck J.S. Bird impact forces and pressures on rigid and compliant targets. Technical Report AFFDL-TR-77-60, Air Force Flight Dynamics Laboratory; 1978.

[4] Hedayati R., Ziaei Rad S. A new bird model and the effect of bird geometry in impacts from various orientations. Aerospace Science and Technology 2013;28:9-20.

[5] Guida M, Marulo F, Meo M, A. Grimaldo, G. Olivares. SPH-Lagrangian study of bird impact on leading edge wing. Composite Structures 2011;93:1060-1071.

[6] Lavoie M, Gakwaya A, Ensan M, Zimcik D. Validation of available approaches for numerical bird strike modelling tools. International Review of Mechanical Engineering 2007.

[7] Heimbs S. Bird strike simulations on composite aircraft structures. Proceedings from SIMULIA Customer Conference 2011.

[8] Goyal V, Huertas C, Vasko T. Smooth particle hydrodynamics for bird-strike analysis using LS-DYNA. American Transactions on Engineering and Applied Sciences 2013;2:84-107.

[9] Kim M, Zammit A, Siddens A, J. Bayandor. An extensive crashworthiness methodology for advanced propulsion systems, Part I: Soft impact damage assessment of composite fan stage assemblies. $49^{\text {th }}$ AIAA Aerospace Sciences meeting including the New Horizons Forum and Aerospace Exposition 2011. 
[10] Liu J, Li Y, Gao X. Bird Strike on a flat plate: Experiments and numerical simulations. International Journal of Impact Engineering 2014;70:21-37.

[11] Hanssen A, Girard Y, Olovsson L, Berstad T, Langseth M. A numerical model for bird strike of aluminium foam based sandwich panels. International Journal of Impact Engineering $2006 ; 32: 1127-1144$.

[12] Liu J, Li Y, Yu X, Tang Z, Gao X, Lv J, Zhang Z. A novel design for reinforcing the aircraft tail leading edge structure against bird strike. International Journal of Impact Engineering 2017;105:89-101.

[13] Smojver I, Ivancevic D. Numerical simulation of bird strike damage prediction in airplane flap structure. Composite Structures; 92(9):2016-2026.

[14] Zhou Y, Sun Y, Huang T, Cai W, SPH-FEM simulation of impacted composite laminates with different layups, Aerospace Science and Technology, 2019;95:105469.

[15] Zhuo Zhang, Liang Li, Dingguo Zhang, Effect of arbitrary yaw/pitch angle in bird strike numerical simulation using SPH method. Aerospace Science and Technology, 2018;81:284293.

[16] Zhou Y, Sun Y, Cai W. Bird-striking damage of rotating laminates using SPH-CDM method, Aerospace Science and Technology, 2019;84:265-27.

[17] Lopez-Lago M, Casado R, Bermudez A, Sernac J. A predictive model for risk assessment on imminent bird strikes on airport areas. Aerospace Science and Technology. 2017;62:19-30.

[18] Martin N.F. Nonlinear finite-element analysis to predict fan-blade damage due to soft body impact. Journal of Propulsion Power 1990;6(4):445-50. 
[19] Heimbs S. Computational methods for bird strike simulation: A review. Computers and Structures 2011;89:2093-2112.

[20] Vedantam K, Bajaj D, Brar N, Hill S. Johnson-Cook strength models for mild and DP 590 steels. AIP Conference Proceedings 2006;845:775.

[21] Hexcel Inc. HexWeb Honeycomb Attributes and Properties Product Catalog

[22] Asprone D, Auricchio F, Menna C, Morganti S, Prota A, Reali A. Statistical finite element analysis of the buckling behaviour of honeycomb structures. Composite Structures 2013;105: 240-255.

[23] Arachchige B, Ghasemnejad H, Augousti A. Theoretical approach to predict transverse impact response of variable-stiffness curved composite plates. Compos Part B 2016;89:34-43.

[24] Performance Composites. Mechanical properties of carbon fibre composite materials 2009.

[25] Shah Q, Topa A. Modelling large deformation and failure of expanded polystyrene crushable foam using LS-DYNA. Modelling and Simulation in Engineering 2014. 
2020-06-15

\section{Effect of bird-strike on sandwich composite aircraft wing leading edge}

Arachchige, B.

Elsevier

Arachchige B, Ghasemnejad H, Yasaee M. (2020) Effect of bird-strike on sandwich composite aircraft wing leading edge, Advances in Engineering Software, Volume 148, October 2020, Article number 102839

10.1016/j.advengsoft.2020.102839

Downloaded from Cranfield Library Services E-Repository 